\title{
¿Qué determina la elección de una Especialidad en Medicina?
}

\author{
Francisco Escobar Rabadána, Jesús López-Torres Hidalgob
}

\begin{abstract}
a Médico de Familia del Centro de Salud Universitario Zona IV de Albacete, Profesor Asociado de la Facultad de Medicina de Albacete

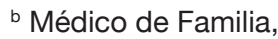
Unidad de Investigación de la Gerencia de Atención Primaria de Albacete, Profesor Asociado de la Facultad de Medicina de Albacete

\section{Correspondencia:}

Francisco Escobar Rabadán. Centro de Salud Universitario Zona IV, C/ Seminario, $\mathrm{n}^{\circ} 4$. Tfno.: 967510094, e-mail: fjescobarr@sescam.jccm.es
\end{abstract}

Recibido el 4 de junio de 2008.

Aceptado para su publicación el 14 de julio de 2008.

\section{RESUMEN}

Existe una gran variabilidad en relación con la proporción de graduados de las diferentes Facultades de Medicina que se decantan por la atención primaria. Se ha desarrollado una importante producción de literatura en relación con la selección de especialidades de atención primaria por parte de los graduados en medicina, interés que parte fundamentalmente de la convicción de que el número de médicos de atención primaria es inadecuado para enfrentar las necesidades de salud (con el objetivo de que al menos un $50 \%$ de los médicos se dedicaran a atención primaria).

Se han formulado diferentes teorías acerca de la toma de decisiones para la elección de carrera en medicina, habiéndose propuesto los antecedentes de los estudiantes, del tipo de habilidad, personalidad y experiencia, como factores que influyen en su elección de carrera. Estos eligen una carrera que satisface sus preferencias y prioridades y es compatible con las restricciones impuestas por sus habilidades y condiciones de vida. Las características demográficas, personalidad y experiencias vitales previas influyen en cómo se enfrentan con la información en el ambiente de la facultad, y esto acaba por determinar la carrera que eligen. Se ha relacionado la preferencia por atención primaria con una orientación biosocial, frente a la biocientífica, al tiempo que se ha subrayado que las actitudes hacia temas de medicina general y la influencia de otras personas tienen el mayor impacto sobre la preferencia de los estudiantes por este nivel asistencial.

La carrera profesional ha sido largamente esperada como un incentivo para ampliar el horizonte profesional y para mejorar la calidad de los servicios prestados a los pacientes (y por consecuencia, para mejorar la salud de la población). Pero no parece que la carrera profesional en la práctica sea la carrera profesional en la teoría, ni que los hechos hayan respondido a las expectativas. La carrera profesional ha sido una oportunidad perdida, pues no va ligada a cambios en el trabajo, ha incrementado las diferencias autonómicas, ha hecho más iguales a los desiguales, ha discriminado a los interinos, y no tendrá impacto en la salud de la población.

Palabras clave: Áreas de Especialidad.

\section{ABSTRACT}

What determines the choice for a specific speciality in Medicine?

There is a wide variability in the proportion of graduates from the different medical faculties that opt to work in Primary Care. Many articles have been published about the selection of primary care specialities by graduates of medicine. The interest in this mainly arises from the conviction that there are an insufficient number of doctors in primary care to meet the health demands (the aim being that at least $50 \%$ of doctors should work in this area).

Different theories have been formulated to explain the decisions taken when choosing a career in medicine and student's background, skills, personality and experience have all been proposed as factors that influence this career choice. Graduates choose a career that satisfies their preferences and priorities and is compatible with the restrictions posed by their abilities and lifestyle. Demographic characteristics, their personality and previous experiences all affect how they process the information they receive in the Faculty, which finally determines the career they choose. A preference for primary care has been related to a biosocial orientation, compared to a bioscientific one, and it has also been shown that attitudes towards general medicine and the influence of other people are the factors with the most impact on the students' preference for this healthcare area.

For a long time the professional career has been expected to act as an incentive to broaden professional horizons and to improve the quality of services offered to the patients (and consequently, to improve the health of the population). However, the real situation differs greatly this theoretical one, and the expectations have not been fulfilled. The professional career is often a lost opportunity, since it is not associated with changes in work, differences between autonomous communities have increased, differences between workers are not taken into account, workers in temporary positions are discriminated against, and it does not have any influence on the health of the population.

Key words. Specialties. 


\section{INTRODUCCIÓN}

Las facultades de medicina varían ampliamente en cuanto a la proporción de sus graduados que se decantan por la atención primaria. En nuestro país hemos de considerar fundamentalmente los graduados cuya opción MIR es medicina de familia, a los que habría que añadir un porcentaje, seguramente pequeño, de aquellos que eligen pediatría.

En la literatura de EEUU cuando se hace referencia a las especialidades de atención primaria se incluye medicina de familia, pediatría y medicina interna generalista, e incluso algunos autores añaden otras especialidades, como geriatría o toco-ginecología. Precisamente en EEUU se ha desarrollado una importante producción de literatura en relación con la selección de especialidades de atención primaria por parte de los graduados en medicina. Este interés parte fundamentalmente de la convicción de que el número de médicos de atención primaria es inadecuado para enfrentar las necesidades de salud del país ${ }^{1}$. La escasez de médicos de atención primaria en este país tiene una especial trascendencia a nivel rural, donde se ha puesto de manifiesto durante la mayor parte del siglo XX. La reciente disminución en el número de médicos que entran en especialidades de atención primaria, junto al hecho de que menos del $4 \%$ de los graduados en facultades de medicina planeen trabajar en áreas rurales y pequeñas ciudades ha aumentado la preocupación por el futuro ${ }^{2,3}$.

En cuanto a la medicina de familia en particular, la disminución del número de graduados de EEUU que eligen esta especialidad ha llevado del $17,3 \%$ en 1997 , a un $10,5 \%$ en 2002 y un $9,2 \%$ en $2003^{4}$. Desde 1977 la American Academy of Family Physicians ha reconocido la necesidad de realizar esfuerzos para aumentar el interés entre los estudiantes de medicina por la medicina de familia como especialidad, y en 1988 se creó la Task Force on Student Interest, cuya meta es que todas las facultades de medicina tengan un departamento de medicina de familia, aumentar el número de graduados que entren a hacer la residencia de medicina de familia, y que se incremente la producción de clínicos cualificados, profesores e investigadores en este campo ${ }^{5}$.

Esta escasez de médicos generalistas se ha puesto de manifiesto también en otros países. En Canadá, la proporción de estudiantes de medicina que eligen medicina de familia como primera opción de residencia ha ido descendiendo, desde un 44\% en 1992 a un $25 \%$ en 2003, quedando plazas vacantes ${ }^{6}$. En Gran Bretaña esta cuestión se ha venido planteando desde los años 60 del siglo XX, debido a una falta de aspirantes a pesar de existir plazas disponibles: solo un $7 \%$ de hombres y un $13 \%$ de mujeres graduados manifiestan su interés por una carrera de medicina general ${ }^{7}$. De igual modo, solo una minoría de graduados australianos muestran interés por carreras de medicina general: alrededor de la cuarta parte eligieron esta opción en 2002, dejando sin cubrir plazas de formación ${ }^{8}$.

Aunque varían las opiniones sobre la razón óptima de médicos de atención primaria y otros especialistas, a mediados de los años 90 el consenso entre los líderes en el mundo de la medicina era que se necesitarían más médicos de atención primaria para asegurar el acceso a una atención de calidad: el objetivo sería que al menos un $50 \%$ de los médicos se dedicaran a atención primaria ${ }^{9}$.

\section{¿QUÉ DETERMINA LA ELECCIÓN DE UNA U OTRA ESPECIALIDAD POR LOS GRADUADOS EN MEDI- CINA?}

Lawson y Hoban ${ }^{9}$ han revisado diferentes teorías que han sido formuladas acerca de la toma de decisiones para la elección de carrera en medicina. Se han propuesto los antecedentes de los estudiantes, del tipo de habilidad, personalidad y experiencia, como factores que influyen en su elección de carrera, proporcionando la base para conocer la naturaleza multifactorial en la toma de decisiones para la elección de carrera médica. Los estudiantes eligen una carrera que satisface sus preferencias y prioridades y es compatible con las restricciones impuestas por sus habilidades y condiciones de vida. Las características demográficas, personalidad y experiencias vitales previas influyen en cómo se enfrentan con la información en el ambiente de la facultad, y esto acaba por determinar la carrera que eligen. Siguiendo otro enfoque, se ha categorizado a los estudiantes según tengan orientación biosocial o biocientífica. Los primeros estarían interesados en trabajar directamente en los problemas psicológicos y sociales de los pacientes. En atención primaria predominaría la orientación biosocial y en las otras especialidades la biocientífica. Sería más probable que los estudiantes elijan atención primaria si tienen una orientación biosocial. Por otra parte, se ha subrayado que las actitudes hacia temas de medicina general y la influencia de otras personas tienen el mayor impacto sobre la preferencia de los estudiantes por atención primaria, mientras que ser varón, tener expectativas de mayores ingresos y tener actitudes positivas hacia la práctica quirúrgica influyen en la elección de una especialidad que no sea atención primaria.

El modelo teórico más citado es el de Bland y Meurer ${ }^{1}$, aunque no ha sido probado empíricamente. Estos autores han desarrollado una teoría por la cual la elección de especialidad contiene tres componentes: factores asociados con características de los estudiantes, tipo de escuela y percepciones de los estudiantes de las características de la especialidad médica. La elección de una carrera por parte de un estudiante se realiza cuando este encuentra aquellas características que mejor cuadran con sus propias necesidades.

Recientemente se ha implicado en la toma de decisiones el "estilo de vida de las especialidades". Dorsey et $\mathrm{al}^{10}$ lo definen por las siguientes características: tiempo personal libre para la práctica de requerimientos de ocio, familia y aspiraciones vocacionales y control de las horas semanales totales empleadas en responsabilidades profesionales, lo que está relacionado con la cantidad de tiempo que queda para actividades independientes de la práctica médica y su reflejo en las horas totales trabajadas y el número de visitas 
nocturnas. Estos autores encuadran 16 especialidades dentro de un "estilo de vida controlable" (anestesia, dermatología, medicina de urgencias, neurología, oftalmología, otorrinolaringología, patología, psiquiatría y radiología) o "estilo de vida incontrolable" (medicina de familia, medicina interna, obstetricia y ginecología, cirugía ortopédica, pediatría, cirugía general y urología), encontrando que de 1996 a 2002 se produjo en EEUU un incremento lineal en el porcentaje de graduados que elegían especialidades con "estilo de vida controlable". Después de controlar por ingresos, horas de trabajo y años de formación postgraduada, el porcentaje de variabilidad que dependía de un "estilo de vida controlable" aumentaba desde un 37 a un 55\%. Como consecuencia se producía una alteración en la distribución de los graduados por especialidad, que para la medicina de familia suponía pasar de un $73 \%$ de puestos de residencia de esta especialidad ocupados en 1996 a un 47\% en 2002.

En un estudio posterior ${ }^{11}$, en base a las respuestas al Cuestionario de Graduación de la Asociación de Colegios Médicos Americanos, estos autores analizan las diferencias por sexos, llegando a la conclusión de que el "estilo de vida controlable" estaba fuertemente asociado con las tendencias en cuanto a elección de especialidad, tanto para hombres como para mujeres. Así, entre 1996 y 2003 la proporción de mujeres que elegían especialidades con un "estilo de vida controlable" aumentaba de 18 a $36 \%$, mientras que para los hombres era del 28 al $45 \%$. En EEUU los estudiantes han creado un acrónimo para referirse a especialidades con "estilo de vida controlable": E-ROAD, correspondiente a emergencias, radiología, oftalmología, anestesia y dermatología.

A conclusiones similares llegaron Lambert y Holmboe ${ }^{12}$ tras estudiar las respuestas al Cuestionario de Graduación de EEUU entre 1990 y 2003. Tanto los hombres como las mujeres demostraban una disminución de su interés por especialidades con "estilo de vida no controlable" de casi el $20 \%$. La popularidad de las especialidades con "estilo de vida controlable" ha estado inversamente relacionada con la popularidad de la atención primaria entre los estudiantes de medicina de EEUU.

Newton et $\mathrm{al}^{13}$ han hablado de un "estilo de vida amable", entendiendo por tal el que permite tiempo libre, oportunidades de disfrutar la vida fuera del trabajo, horas de trabajo predecibles, tiempo para desarrollar actividades fuera del trabajo y tiempo familiar. Estos autores analizan las preferencias de especialidad y las preocupaciones por ingresos y estilo de vida de 1.334 estudiantes de cuarto curso de dos facultades entre 1998 y 2004. Eran los propios estudiantes los que decidían el estilo de vida de cada especialidad, mostrando en sus percepciones un continuum desde un "estilo de vida amable" (radiología, rehabilitación, medicina de urgencias, oftalmología, anestesia, urología, dermatología y otorrinolaringología) a "nada amable" (cirugía general, obstetricia-ginecología). Contrariamente a los estudios anteriores, las respuestas de los estudiantes encuadraban las especialidades de atención primaria como con un "estilo de vida intermedio". Tanto el estilo de vida como los ingresos influían crecientemente en la elección de carreras por los estudiantes durante el periodo de estudio. Sin embargo, la preocupación por el estilo de vida y los ingresos no caracterizaban a todas las especialidades, encontrando que los estudiantes que elegían ciertas especialidades (radiología, rehabilitación, medicina de urgencias, oftalmología, psiquiatría, medicina de familia y pediatría general) era más probable que valoraran el estilo de vida que los ingresos, mientras que otros (los que elegían subespecialidades de medicina interna, ortopedia, cirugía general y obstetricia-ginecología) era más probable que valorar los ingresos sobre el estilo de vida.

Centrándonos en las especialidades de atención primaria, ¿podemos establecer una relación entre la elección de las mismas y determinados factores?

Siguiendo el esquema propuesto por Bland, Meurer y Maldonado ${ }^{1}$, en su revisión de las evidencias de alta calidad generadas en este campo entre 1987 y 1993 en EEUU y Canadá, vamos a revisar los distintos determinantes e influencias en la elección de especialidad.

\section{Tipo de facultad, misión y estructura.}

Como han puesto de manifiesto Block et $\mathrm{al}^{14}$ (1998), la facultad tiene un papel socializante que influye en las actitudes de los estudiantes hacia la elección de carreras de atención primaria. Las características de la facultad tienen un impacto sobre los resultados de la educación médica: si ésta es pública o privada, la presencia y tamaño de un departamento de medicina de familia, intensidad de la investigación, tamaño de las clases o la antigüedad de la facultad. Los estudiantes de una facultad particular tienen puntos de vista más similares del grado en que la atención primaria es promovida, que estudiantes de facultades diferentes. Sin embargo las expectativas de los estudiantes dependen sobre todo de la especialidad deseada por ellos: los estudiantes que se inclinan más hacia carreras de atención primaria demuestran puntos de vista más positivos hacia la atención primaria. Estos autores demuestran que, a pesar de esto, el predictor más fuerte del estímulo para entrar en atención primaria es el registro histórico de la facultad en cuanto a producción de médicos de familia. Así pues, la cultura de aquellas facultades con una fuerte misión relativa a atención primaria anima a los estudiantes a hacerse generalistas.

Whitcomb et al ${ }^{15}$ compararon entre los años 1981 y 1985 un grupo de 25 facultades que producían altos porcentajes de médicos de atención primaria (del 35 al $56 \%$ ) y otro de 25 con bajos porcentajes (22-29\%). Consideran que la marcada diferencia en la producción de médicos de atención primaria podría ser parcialmente explicada por el impacto que la reputación de las facultades podría haber tenido sobre las decisiones de los estudiantes acerca de a qué facultad acudir, si bien dan más importancia al ambiente educacional de la facultad. En una línea de investigación similar, Campos-Outcalt et $\mathrm{al}^{16}$ compararon las respuestas a un cuestionario de graduados de los años 1997 a 1999 
de 24 facultades (la mitad de ellas con una producción creciente de médicos de familia y el resto con una producción decreciente), en función de que entraran a hacer una residencia de medicina de familia o una residencia de otra especialidad del ámbito de la atención primaria, encontrando que las variables relacionadas con acudir a uno u otro grupo de facultades eran el número de rotaciones clínicas en medicina de familia y atención primaria requeridas, la percepción de la competencia clínica de los profesores de medicina de familia y un expectativa de practicar en un área rural.

En relación con la titularidad de la facultad, Pugno et $\mathrm{al}^{5}$ han demostrado que los graduados de las facultades de propiedad pública tienen una probabilidad casi 2 veces mayor de ser residentes de medicina de familia que los de las privadas (16,2\% frente a 9,3\%). Esta es una de las características estructurales que mejor predice la elección de una especialidad de atención primaria, junto a la antigüedad de la facultad, existiendo una correlación negativa entre ésta y la producción de generalistas. Esta correlación negativa se daba también con relación al tamaño de las clases ${ }^{17}$. En cuanto a la cantidad de fondos públicos recibidos por la facultad para investigación, existe una asociación inversa con la producción de médicos de familia, así como de médicos rurales y generalistas ${ }^{18}$.

\section{Composición del profesorado.}

Es probable que las características de la facultad afecten en gran medida la composición del profesorado, siendo estos quienes toman las decisiones acerca de quienes son admitidos a la facultad, qué currículum y qué percepciones acerca de las diferentes especialidades adquieren los estudiantes. La mayoría de los profesores en las facultades de medicina no tienen experiencia personal ni entrenamiento en atención primaria, de forma que el porcentaje de profesores que sean de atención primaria puede jugar un papel fundamental.

Comentarios negativos acerca de la medicina de familia por parte del profesorado, especialmente de otras especialidades, pueden disuadir a muchos estudiantes inicialmente interesados en la misma. Así lo ponen de manifiesto Hearst et $\mathrm{al}^{19}$ al analizar las respuestas de 138 estudiantes que habían participado en actividades relacionadas con la medicina de familia. Alrededor de la tercera parte de los estudiantes de primer curso habían recibido cualquier tipo de feedback negativo, aumentando en los cursos sucesivos de forma que en cuarto casi todos lo habían recibido. Los estudiantes referían haber escuchado comentarios sobre los médicos de familia que les calificaban de incompetentes o no bien entrenados, o la idea de que si hay que saber mucho hay más riesgo de errores. Curiosamente los comentarios más frecuentes ("tú no puedes saber de todo", "tú eres demasiado inteligente como para ser médico de familia") plantean una contradicción. Los autores recomiendan que se aumente el contacto de los estudiantes con médicos de familia en los últimos cursos, cuando se toman las decisiones finales sobre la residencia.
Por el contrario, se ha demostrado que el contacto temprano de los estudiantes con profesores médicos de familia no se asocia con una mayor selección de la residencia de medicina de familia ${ }^{20,21}$. En estas circunstancias su papel puede estar relacionado, no tanto con la influencia directa sobre los estudiantes, sino por la que tengan sobre otros profesores, que podrán tener un conocimiento más cercano de sus colegas.

La influencia de un profesor consejero puede ser un factor determinante en la decisión de los estudiantes para elegir atención primaria. Así se pone de manifiesto en el estudio realizado por Osborn ${ }^{22}$ a partir de las respuestas a un cuestionario postal de 102 estudiantes de la facultad de medicina de la Universidad de California en los meses previos a su graduación en el año 1992. El 77\% de los estudiantes decían haber tomado la decisión sobre sus especialidades en los dos últimos cursos; 50 elegían especialidades de atención primaria.

La existencia de departamentos de medicina de familia en las facultades se ha asociado con una mayor probabilidad de que sus graduados entren en programas de residencia de esta especialidad. En EEUU, en 1999, el $14,3 \%$ de los graduados de facultades con departamentos de medicina de familia estaban en primer año de residencia de esta especialidad, frente al $8,8 \%$ de los de facultades con divisiones de medicina de familia y el $1,9 \%$ de los de aquellas facultades sin estructura administrativa para la misma ${ }^{5}$.

Sin embargo, Campos-Outcalt y Senf ${ }^{23}$ no encuentran esta asociación al comparar las proporciones medias de estudiantes que entraban en programas de residencia de medicina de familia 3 años antes y después del establecimiento de un departamento en facultades de EEUU entre 1984 y 1993. Aún así, estos autores consideran que la presencia de un departamento es un primer paso importante para alcanzar otras iniciativas que si pueden contribuir a aumentar la proporción de estudiantes que elijen medicina de familia, como tiempo requerido de rotación, matricular más estudiantes interesados por la medicina de familia, y el aumento del número, prestigio e influencia de la misma en la facultad.

\section{Admisión.}

Algunas facultades favorecen la entrada de estudiantes con un cierto perfil, que pueda hacerles más proclives a elegir carreras de atención primaria.

Martini et a ${ }^{17}$ encontraron que un $40 \%$ de las facultades de EEUU con alta producción de generalistas (cuartil superior) daba preferencia de admisión a solicitantes que afirmaran un interés por la práctica generalista, mientras que en las de producción intermedia o baja las proporciones eran del 19 y $3 \%$, respectivamente. Por otra parte un $42 \%$ de las facultades daban preferencia de admisión a estudiantes de áreas rurales, existiendo una correlación positiva entre dar esta preferencia y la producción de generalistas.

Se ha sugerido que la admisión de estudiantes que afirman un interés por la medicina de familia es más 
útil para aumentar el número de graduados que entran en residencias de esta especialidad que intentar persuadir a los estudiantes para que la seleccionen después de su admisión en la facultad. Así se pone de relieve en el trabajo realizado por Bowman et $\mathrm{al}^{24}$ a partir de las respuestas a diversos cuestionarios pasados por los colegios médicos americanos, que recogían datos de 30.789 estudiantes que se graduaron en facultades de medicina de EEUU en 1991 y 1992. El 3,3\% de los estudiantes "mantenían" un interés inicial por la medicina de familia (es decir, originalmente planeaban entrar en esta especialidad y en el año de postgrado estaban haciendo esta residencia); el 6,4\% habían sido "ganados" (originalmente pensaban en hacer una especialidad distinta, pero entraban en una residencia de medicina de familia); 6,3\% "perdían interés" (originalmente se identificaban con medicina de familia, pero entraban en otra residencia, que en $2 / 3$ de los casos no era de atención primaria); 70,1\% se consideraban "nunca interesados" (no habían expresado interés por la medicina de familia ni entraban a hacer esta residencia); y el 13,9 restante tenían datos incompletos. Ahora bien, de aquellos estudiantes que estaban originalmente interesados por la medicina de familia, 34,5\% entraban a hacer la residencia en esta especialidad, mientras que solo lo hacían el 8,3\% de los no interesados originalmente.

Los cuatro grupos de estudiantes descritos por estos autores diferían en sus características demográficas, de actitudes y de experiencias. Las mujeres era más probable que estuvieran interesadas en la medicina de familia en la matriculación, pero también era más probable que perdieran el interés a lo largo de los años de facultad. Los graduados que entraban en residencia de medicina de familia era más probable que hubieran decidido hacerse médicos a una edad más tardía, que procedieran de áreas rurales y que tuvieran una más baja expectativa de ingresos; el énfasis sobre la atención primaria y la prevención y la práctica en pequeñas comunidades eran más importantes para los futuros médicos de familia. Por el contrario, el prestigio, los ingresos, la preocupación por la incertidumbre en el diagnóstico y el tratamiento, el contenido intelectual de la especialidad y las oportunidades para la investigación eran más importantes para los futuros especialistas en otras áreas.

\section{Currículum.}

La existencia de asignaturas relacionadas con la atención primaria, y/o rotaciones clínicas en este nivel asistencial ha sido relacionada con un mayor interés por especialidades de atención primaria. Sin embargo, como señalábamos anteriormente, no se ha demostrado una mayor probabilidad de elegir una carrera de atención primaria y el haber realizado cursos específicos en los dos primeros años de carrera ${ }^{20,21}$. Por el contrario, sí que se ha encontrado asociación entre la presencia de rotaciones de medicina de familia y la proporción de estudiantes que eligen esta especialidad.

Campos-Outcalt y $\operatorname{Senf}^{23}$ han demostrado que en EEUU la introducción de una rotación obligatoria en medicina de familia en el tercer curso tenía un efecto positivo sobre la proporción de estudiantes que elegían medicina de familia como especialidad: $2,36 \%$ en las públicas y $2,07 \%$ en las privadas. Las facultades que tenían la rotación obligatoria durante más tiempo tenían proporciones más altas de estudiantes que entraban en medicina de familia.

Solo el $6 \%$ de los médicos generalistas que participaron en el estudio de Martini et al $^{17}$ reconocía haber realizado su elección de especialidad durante los primeros 2 años de la facultad, mientras que el $40 \%$ elegían su especialidad en los años de rotación clínica. Un 42\% consideraba las rotaciones experiencias que les habían influido fuertemente en su elección de especialidad. Una rotación rural obligatoria tenía también una correlación positiva con la proporción de generalistas.

No está claro cómo el entrenamiento clínico en medicina de familia afecta a la elección de especialidad de los estudiantes. Podría ser por el entrenamiento en sí mismo o la exposición a la especialidad, aunque el tiempo requerido para esta formación podría estar reflejando la fuerza de la medicina de familia en la facultad. En cualquier caso, las preferencias de los estudiantes por la especialidad cambian tras realizar la rotación en medicina de familia, aunque estos cambios no siempre son predictivos de la elección de especialidad última ${ }^{18}$.

En cuanto a la existencia de una formación longitudinal a lo largo de la carrera, Herold et $\mathrm{al}^{25}$ estudiaron la influencia sobre la elección de especialidad de un programa de formación en atención primaria a lo largo de los 4 años de los estudios de medicina. De 201 estudiantes que se presentaron voluntarios, fueron seleccionados al azar 93 para participar en el programa. Era significativamente más probable que los voluntarios, independientemente de que hubieran participado o no en el programa, eligieran especialidades de atención primaria, mientras que los no voluntarios optaban por especialidades de alta tecnología. Concluyen que el programa no parece influir la elección de especialidad de los estudiantes, porque los interesados en participar, independientemente de que lo hagan o no, era más probable que eligieran especialidades de atención primaria.

\section{Características y valores de los estudiantes al ingreso.}

Se han propuesto diferentes circunstancias que podrían inclinar la decisión de los estudiantes hacia una u otra especialidad. Revisaremos las más importantes:

Sexo: las mujeres y los hombres difieren en sus patrones de elección de especialidad, aunque cambian con el tiempo. Diferentes estudios han demostrado una tendencia significativa en cuanto a que las mujeres elijan medicina de familia, pediatría y medicina interna general. Los motivos para entrar en la facultad de medicina y para elegir especialidad difieren según el sexo: las mujeres valoran más la relación con los pacientes y colegas, y menos los ingresos y el prestigio. 
Edad y estado civil: tener más edad en el momento de la matriculación, estar casado y tener niños tiene una asociación positiva con la elección de una especialidad de atención primaria, que podría tener relación con la preferencia por programas de residencia más cortos.

- $\quad$ Origen socioeconómico: en USA se ha encontrado asociación con este factor, debiendo tener en cuenta además la relación existente entre minorías étnicas y más bajo nivel socioeconómico. Estos estudiantes pueden tener acumuladas deudas en relación con la financiación de sus estudios. Por otra parte es más probable que estén interesados en ejercer en áreas socioeconómicamente deprimidas. Los estudiantes hijos de médicos tienden a elegir especialidades que no son de atención primaria, lo que puede ser reflejo del estatus socioeconómico, un mayor conocimiento de la especialidad, el estatus relativo de la atención primaria dentro de la comunidad médica o el apoyo familiar dado a la elección.

- $\quad$ Origen geográfico: estudiantes de pequeñas ciudades o de origen rural es más probable que elijan atención primaria.

- $\quad$ Antecedentes académicos: no en cuanto a una relación con el rendimiento académico previo, sino en cuanto a que los estudiantes con una formación de "base amplia", con actividades extracurriculares, es más probable que elijan especialidades con alto nivel de interacción médico-paciente, como la medicina de familia.

- Personalidad: no se ha demostrado su valor como predictor en la elección de especialidad, probablemente porque los instrumentos existentes no son capaces de establecer diferencias entre ellos. Los estudiantes de medicina son un grupo homogéneo: con relación a la población general son en conjunto más competitivos, académicamente superespecializados, muy cumplidores, altamente motivados y disciplinados.

Expectativas de la carrera al matricularse: las expectativas de los estudiantes dependen sobre todo de la especialidad deseada por ellos: los estudiantes que se inclinan más hacia carreras de atención primaria demuestran puntos de vista más positivos hacia la atención primaria ${ }^{14}$.

Por otra parte, la decisión de realizar la residencia en atención primaria puede venir determinada por otros factores, como la actitud del estudiante hacia el sistema de atención a la salud. Lawson et al ${ }^{26}$ realizaron un análisis de regresión logística en el que las variables independientes eran las características de los estudiantes del modelo de Bland-Meurer, y la variable dependiente era la elección de residencia de atención primaria. Participaron en el estudio 555 estudiantes, de los que el $51 \%$ elegía estas especialidades. El modelo que mejor predecía la elección de una residencia de atención primaria incluía las variables: ser mujer, obtener puntuaciones bajas en las rotaciones de psiquiatría y cirugía y altas puntuaciones en la rotación de medicina interna, decidir no participar en un proyecto de investigación, estar en desacuerdo con que "los cambios en el sistema de atención a la salud tengan un efecto negativo sobre los médicos", y de acuerdo con que "el acceso a la atención médica y a la prevención de la enfermedad es un problema en EEUU", y planear ejercer en áreas infradotadas.

Martini et a 17 encontraron que los "valores sociales personales", en el sentido de responsabilidad social y compromiso de servicio, eran el factor que los médicos generalistas reconocían como mayor influencia en su elección de carrera: casi el $67 \%$ los consideraba una fuerte o muy fuerte influencia en su elección de una especialidad generalista.

Kassebaum et $\mathrm{a}^{27}$, a través del análisis por regresión logística de la información obtenida de bases de datos de la Asociación de Colegios Médicos Americanos, estudiaron las intenciones de los graduados en 1995 en EEUU para seguir carreras generalistas. En particular, los factores que se asociaban positivamente con la elección de medicina de familia eran el sexo femenino, una edad mayor o igual a 28 años, un origen rural y la presencia de un departamento de medicina de familia. Sin embargo, existían dos variables especialmente poderosas: una probabilidad casi cinco veces mayor cuando los estudiantes habían indicado una preferencia por la medicina de familia al matricularse y una probabilidad de más de 9 veces cuando los estudiantes seguían una asignatura optativa de medicina de familia. Por el contrario, eran influencias negativas los intereses en altas posibilidades de ingresos e investigación.

En un estudio realizado en Canadá, Wright et $\mathrm{al}^{6}$ identificaron una serie de factores y de características demográficas asociados con la perspectiva de los estudiantes de dedicarse a la medicina de familia. En base a las respuestas a un cuestionario de 519 estudiantes en las dos primeras semanas de estancia en la facultad establecieron sus tres primeras opciones de carrera y el grado en que 25 variables contribuían (escala Likert de 5 puntos, donde el 1 correspondía a la ausencia de influencia y el 5 a la máxima influencia) a la elección de la primera opción. El 19,5\% consideraba la medicina de familia su primera opción, y un $53 \%$ la incluía en alguna de las tres posiciones. La media de edad de los que elegían medicina de familia era significativamente mayor que la de aquellos que elegían otras especialidades: 26 (DE: 5,1 ) vs 23,7 años (DE: 2,9). Las mujeres elegían medicina de familia como primera opción más frecuentemente que los hombres ( $23 \%$ vs $16 \%, p<0,05)$. En el análisis factorial sobre las respuestas a la primera elección de carrera identificaron 5 factores que incluían 17 de las 25 variables y explicaban el $52 \%$ de la varianza en las respuestas. Un análisis de regresión logística revelaba que la mayor orientación social, el deseo de un ámbito variado de práctica, la menor orientación hospitalaria, la mayor edad, la preocupación por el estilo de vida médico y la menor población de la comunidad donde el estudiante 
había completado su formación secundaria eran, en este orden de importancia, predictivos de la elección de medicina de familia como primera opción.

\section{Factores que afectan a los valores de los gra- duados.}

Circunstancias que acontecen durante los años de facultad pueden cambiar los valores o necesidades de los estudiantes:

- Endeudamiento. La relación con la elección de especialidad puede venir determinada por dos efectos distintos: por una parte puede llevar a seleccionar especialidades con mayores ingresos, pero también puede llevar a seleccionar especialidades de menor duración para evitar el incremento de las deudas. Rosenblatt y Andrilla ${ }^{28}$, utilizando las respuestas sobre deudas y elección de carreras del Cuestionario de Graduación de la Asociación Médica Americana en 2002 , encontraron que un $83,5 \%$ de los estudiantes graduados tenía deudas, con una media por estudiante de 86.870 \$. Los estudiantes admitían que los altos niveles de deudas influían en sus elecciones de carrera, existiendo una relación inversa entre el nivel total de deudas educacionales y la intención de entrar en atención primaria. Sin embargo, su efecto era modesto cuando se tomaban en consideración otros factores, como los demográficos. Así, por ejemplo, las mujeres estaban mucho más interesadas en atención primaria y pediatría que los hombres.

- $\quad$ Socialización. La educación médica influye en las actitudes y la personalidad de los estudiantes. Las normas y la cultura del entorno de la facultad pueden jugar un papel significativo conformando los valores de los estudiantes de medicina. A lo largo de los años de facultad los estudiantes experimentan una disminución de la orientación humanística y un aumento en el hedonismo. Al final de la carrera es mayor la orientación a la profesión y las condiciones de trabajo, y menos hacia el cuidado de los pacientes, en comparación con los estudiantes de primer año. Los estudiantes que elijen atención primaria tendrían una mayor orientación al cuidado de los pacientes que los que elijen otras especialidades.

Como pusieron de manifiesto Lynch et $\mathrm{al}^{29}$, las percepciones positivas hacia la atención primaria de los estudiantes al inicio de su formación pueden cambiar conforme van desarrollando a lo largo de los años de facultad percepciones realistas acerca de las demandas profesionales sobre los médicos de atención primaria.

Ya hemos señalado la frecuencia con que los estudiantes de medicina escuchan comentarios negativos acerca de la medicina de familia, especialmente por parte de profesores de otras especialidades, del tipo "los médicos de familia no pueden dominar el contenido de la especialidad" o "no son tan inteligentes como otros médicos" ${ }^{30}$.

Zinn et $a^{31}$ ratifican esta apreciación en el sentido de que durante el curso de su educación y entrenamien- to los estudiantes experimentan una erosión de sus orientaciones hacia la atención primaria conforme van progresando a través de la facultad de medicina y hacia la residencia. Se basaron en las respuestas a una entrevista telefónica dirigida a muestras probabilísticas estratificadas de estudiantes de medicina y residentes de EEUU en 1994 y 1997, en la que preguntaban por sus actitudes en relación con enfrentarse a temas psicosociales y su percepción de las actitudes de los profesores y sus compañeros hacia la atención primaria, comparando las respuestas a lo largo del tiempo y entre grupos. Entre el primer y el cuarto curso de la facultad de medicina había una disminución a lo largo del tiempo de la orientación de los estudiantes hacia aspectos socioemocionales de la atención a los pacientes $(61,6 \%$ vs $42,7 \%, p=0,001)$ y de sus percepciones de que trabajar con temas psicosociales de los pacientes hacía a la atención primaria más atractiva $(56,3 \%$ vs $43,5 \%, p=0,01)$. Este patrón se mantenía en los residentes entrevistados en 1997, quienes era incluso menos probable que dijeran que el tratar temas psicosociales hacía a la atención primaria más atractiva (26,9\%). Para los estudiantes de cuarto de 1994 que eran residentes de tercer año en 1997 había una percepción aumentada de que los médicos y profesores de otras especialidades tenían actitudes positivas hacia la atención primaria $(20,8 \%$ vs $33,0 \%$, $p=0,005 ; 28,3 \%$ vs $45,7 \%, p<0,001$, respectivamente), lo que reflejaría una percepción de que el entorno para la atención primaria en el ámbito académico había mejorado con el tiempo.

Las experiencias vividas por los estudiantes en atención primaria pueden influir en un sentido positivo, ya que el mayor conocimiento de las especialidades y las experiencias clínicas positivas a lo largo de los años de facultad determinan cambios en las preferencias de los estudiantes. En un estudio realizado por Market ${ }^{32}$, a partir de la información recopilada de 815 graduados de la Wright State University School of Medicine entre 1981 y 1990 , se encontró que 217 de ellos cambiaban sus preferencias durante los años de facultad. En concreto, 53 se inclinaban hacia atención primaria, y 164 a otras especialidades. Las razones relativas a la experiencia en la facultad tenían más influencia que razones de tipo personal o social, y en especial más para los que cambiaban a otras especialidades que para aquéllos que cambiaban a atención primaria. La razón más frecuente para cambiar era el tener un mayor conocimiento de las especialidades: $62 \%$ de los que cambiaban a atención primaria y $81 \%$ de los que cambiaban a otras especialidades. La segunda respuesta elegida, por ambos grupos, era el haber tenido una experiencia positiva en una rotación clínica en una determinada especialidad. Entre las razones seleccionadas con menos frecuencia, era más probable que fueran citadas por los que se cambiaban a atención primaria el contenido y el énfasis del currículum (19\% vs $7 \%$ ) y la localización de la residencia ( $17 \%$ vs $6 \%)$, mientras que para los que cambiaban a otras especialidades era más importante el tener mejores oportunidades financieras ( $17 \%$ vs $4 \%$ ). Los que cambiaban a atención primaria las razones que esgrimían eran: 
consideraciones familiares o personales, influencia positiva de profesores específicos en la especialidad elegida, experiencias negativas en una rotación clínica de otra especialidad y la necesidad social de médicos. Los que se cambiaban a otras especialidades citaban: influencia positiva de profesores específicos en las especialidades elegidas, experiencias negativas en una rotación clínica, consideraciones familiares y/o personales y mayor estímulo intelectual. Un $23 \%$ de los que cambiaban sus preferencias argumentaba opiniones negativas de profesores de otras especialidades.

Estos cambios en las preferencias por una determinada especialidad fueron también estudiados por Schafer et $\mathrm{al}^{33}$, en base a las respuestas de 320 estudiantes de la Universidad de California, San Francisco, después del Nacional Resident Matching Program Match y antes de su graduación en los años 1996,1997 y 1998. De 41 que decían que la medicina de familia había sido su primera opción de especialidad previamente a comenzar las rotaciones clínicas, solo 15 (37\%) acababa eligiéndola para su residencia; en comparación, los porcentajes para medicina interna y pediatría eran del $50 \%$ y $69 \%$. En relación con los que rechazaban otras especialidades, los estudiantes que rechazaban medicina de familia era más probable que citaran como razones el insuficiente prestigio, el bajo contenido intelectual y la preocupación acerca de una maestría demasiado extensa en cuanto a las áreas de contenido. Los estudiantes reconocían que sus compañeros, los residentes y los profesores a menudo hablaban mal de la medicina de familia.

Nieman et $\mathrm{al}^{34}$ recopilaron información de 10.081 graduados entre los años 1992 y 2000 en facultades de medicina de Texas, comparando la proporción de los que elegían medicina de familia u otras residencias de atención primaria, según hubieran o no participado en un programa de tutorización en medicina de familia. El $27,9 \%$ de los participantes elegían medicina de familia, frente al $15,7 \%$ de los que no habían participado.

El efecto positivo de la experiencia en una determinada especialidad puede ser transitorio, como se demuestra en el estudio de Morrison y Murray ${ }^{35}$. Estos autores describieron los factores que influían en la elección de carrera de estudiantes de medicina de último curso de la Universidad de Glasgow, especialmente el efecto de un rotatorio de 4 semanas en medicina general, y los cambios en las elecciones de carrera entre el final del curso y el final del año de prerregistro de residencia. El estudio consistió en la administración de un cuestionario a 206 estudiantes de medicina que iban a realizar su rotatorio clínico final, antes e inmediatamente después de las 4 semanas que duraba, y el posterior envío de un cuestionario postal después del año de prerregistro de residencia (16 a 26 meses después del segundo cuestionario). Los 3 cuestionarios fueron completados por 131 estudiantes. Antes del rotatorio de medicina general, era más probable que las mujeres consideraran la medicina general su primera opción de carrera (era la primera elección para el 36,6\% de las mujeres frente al 8,3\% de los hombres), sin embargo no había diferencias por sexos después del rotatorio.
En ese momento la medicina general había pasado de ser considerada la materia del curso que más les gustaba para solo el $4 \%$ a el $47,1 \%$ de los estudiantes, mientras que el número de los que afirmaban que era probable o muy probable que eligieran medicina general aumentaba de 60 a 72 (principalmente por el cambio de opción de estudiantes varones), sin embargo en el tercer cuestionario habían descendido a 56. Concluyen así que el rotatorio de medicina general influía a los estudiantes, especialmente varones, hacia una carrera de medicina general, pero este efecto era transitorio.

\section{Necesidades que satisfacer.}

Los estudiantes que prefieren especialidades de atención primaria tienen un mayor deseo de contacto con los pacientes y están más interesados en servir a poblaciones diversas, tratar una variedad de problemas médicos y realizar cuidados de salud con los pacientes. Estos estudiantes están menos preocupados con practicar una medicina hospitalaria, realizar cirugía, temas de malpraxis, ingresos y prestigio, que aquellos que prefieren otras especialidades.

Fincher et $\mathrm{al}^{136}$ analizaron las posibles influencias sobre la elección de especialidad en base a las repuestas a un cuestionario de 404 graduados de la facultad de medicina del Medical College of Georgia en los años 1988, 1989 y 1990. Los estudiantes que elegían especialidades de atención primaria (42\%) estaban influidos positivamente más a menudo por su deseo de mantener opciones abiertas para una futura subespecilización ( $85 \%$ vs $58 \%$ ) y su deseo de oportunidades de atención longitudinal a los pacientes (95\% vs 54\%). Aquellos que elegían especialidades que no eran de atención primaria estaban influidos más a menudo por su deseo de recompensas económicas (69\% vs $35 \%$ ) y sus percepciones de estilo de vida tras la residencia (74\% vs $60 \%$ ) y prestigio de la especialidad $(57 \%$ vs $36 \%)$. En un trabajo posterior ${ }^{37}$, basado en las respuestas a un cuestionario enviado por correo a graduados de 9 facultades en 1991, confirman algunos de estos hallazgos. Mostraron un interés por hacer una residencia de atención primaria el $45 \%$ de los 803 participantes. Los factores que se asociaban positivamente con la elección de una especialidad de atención primaria, cuando se controlaba por otros factores, eran el deseo de proporcionar una asistencia integral, mantener opciones abiertas y prestar atención ambulatoria, mientras que el deseo para recompensa económica estaba asociado negativamente con la elección de una especialidad de atención primaria.

En el ya mencionado trabajo de Osborn ${ }^{22}$, los factores que se asociaban con la elección de una especialidad que no fuera de atención primaria eran las expectativas de futuros ingresos y las oportunidades de trabajo con nuevas tecnologías.

\section{Percepción de las especialidades.}

Una de las mayores dificultades que tienen los estudiantes para elegir especialidad es la falta de información sobre las mismas. La mayoría de los estudiantes aprenden acerca de las especialidades durante sus años de facultad participando en cursos y a través de 
la observación de los profesores, residentes y otros estudiantes. Si las experiencias no reflejan la realidad, la percepción de los estudiantes puede estar distorsionada.

La mayoría de los estudiantes no tienen definida la especialidad que van a hacer cuando entran en la facultad de medicina. A lo largo de la carrera van variando las preferencias. El mayor interés por una especialidad se da, como ya hemos comentado, inmediatamente después de una rotación. Muchos estudiantes que tienen preferencia por especialidades de atención primaria al inicio de su formación, cambian su perspectiva durante las rotaciones clínicas.

En base a las respuestas a un cuestionario, enviado por correo electrónico, de 479 estudiantes de medicina de todos los cursos en la Facultad de Medicina de Londres en el año 2001, Ali y Jones ${ }^{7}$ concluyeron que el número de estudiantes que expresan una preferencia temprana por la medicina general es bajo $(13,8 \%)$, si bien un $23,8 \%$ estaban inseguros sobre su futura carrera. La medicina general era más popular entre las mujeres (32,1\% vs $10,5 \%)$, existiendo una preferencia aumentada por la misma en aquellos estudiantes que habían recibido formación específica en este campo (22\% vs 9,6\%).

En un estudio cualitativo con grupos focales, llevado a cabo por Tolhurst y Stewart ${ }^{8}$, para conocer los factores que influyen para que un estudiante de medicina se decante por medicina general, y en el que participaron 82 estudiantes de primer y último año de las facultades de medicina australianas en 2002, de los que alrededor de la mitad estaban interesados en la misma, encontraron los siguientes factores atrayentes: naturaleza del trabajo (incluyendo su diversidad), continuidad de la atención, contexto comunitario y condiciones de trabajo (incluyendo flexibilidad de formación y trabajo, disponibilidad de parte del tiempo de trabajo y elasticidad en las cualificaciones). Como factores negativos destacaban la amplitud del conocimiento necesario, trabajo aburrido (en medicina general urbana), sobrecarga burocrática, tener que llevar un negocio y condiciones de trabajo (incluyendo una relativamente pobre remuneración, carga de trabajo en medicina general rural y pobre estatus de los médicos generales). La mayoría de los participantes de primer año tenían ideas poco definidas acerca de sus futuras intenciones de carrera, aunque muchos anticipaban que sus intereses podrían verse influidos por su experiencia en el pregrado. Por su parte, los estudiantes de último año recordaban que sus experiencias en el pregrado habían influido en sus preferencias de carrera, recordando algunos de ellos las actitudes negativas hacia la medicina general que habían encontrado en otros especialistas, incluyendo profesores.

Los factores relacionados con la elección de especialidad de los médicos de familia tienen similitudes con los de otras especialidades también relacionadas con la atención primaria. Senf et $\mathrm{al}^{38}$ enviaron por correo un cuestionario a todos los médicos de familia y un número igual de otros médicos de atención primaria graduados en 24 facultades de medicina de EEUU en 1997 a 1999, 12 de las cuales habían aumentado la proporción de graduados que elegían medicina de familia, mientras que las otras la habían disminuido. De 2985 cuestionarios enviados recibieron 1457, oscilando la proporción de respuestas según las facultades (33-73\%), y según especialidades (médicos de familia: $57,6 \%$; combinación de medicina interna y pediatría: 56,3\%; pediatría: 50,4\%; medicina interna: 38,3\%). Para los médicos de familia, el factor más importante era "las relaciones con los pacientes", seguido por "querer un enfoque hacia la práctica de la medicina similar a la de los médicos de familia". Los internistas indicaban como más importante "querer trabajar con adultos", seguido por "un enfoque de medicina interna a su práctica". Lo más importante para los pediatras era "trabajar con niños", seguido por "la relación con los pacientes como otro pediatra". Aquellos que combinaban medicina interna y pediatría indicaban más a menudo "un deseo de trabajar con niños", seguido por "un enfoque a la medicina como otros de su especialidad". Así pues, las razones para elegir especialidad estaban relacionadas con la congruencia entre el graduado y los médicos en la especialidad de su preferencia o el proceso de prestar atención en la misma. Se pone de manifiesto la existencia de un modelo de rol, relacionado con el conocimiento de los estudiantes del encaje entre ellos mismos y la especialidad que han elegido, siendo éste más marcado para los médicos de familia que para las otras especialidades.

Para concluir esta revisión, queremos recordar las recomendaciones que Bland, Meurer y Maldonado ${ }^{1}$ proponen para aumentar la incorporación de estudiantes a los programas de residencia de atención primaria:

1. Desarrollar departamentos académicamente creíbles de medicina de familia (en su ámbito también medicina interna general y pediatría general).

2. Cambiar la cultura de las facultades para valorar la atención primaria.

3. Cambiar las políticas de admisión a favor de estudiantes interesados en atención primaria y dar peso a características de los estudiantes que pudieran predecir una futura elección de carreras de atención primaria.

4. Cambiar la composición de los comités de admisión para incluir personas que sean útiles en la selección de estudiantes que acaben en carreras de atención primaria.

5. Usar procesos de reclutamiento y selección que tengan mayores probabilidades de atraer a estudiantes que elijan carreras de atención primaria.

6. Tener profesores de atención primaria clínicos, además de contar con voluntarios como profesores de prácticas en los cursos iniciales.

7. Establecer experiencias de atención primaria longitudinales obligatorias.

8. Establecer rotaciones de medicina de familia obligatorias.

9. Establecer un curso sobre necesidades de salud de la sociedad y el papel de los médicos.

10. Establecer un programa de consejo sobre carreras, incluyendo información formal sobre las características clave de las especialidades.

11. Estimular a las facultades a evaluar de forma continuada el impacto de sus programas. 
12. Requerir de los poderes públicos inversiones para la formación y la investigación en atención primaria, así como para el estudio de la educación médica.

\section{BIBLIOGRAFÍA}

1. Bland CJ, Meurer LN, Maldonado G. Determinants of primary care specialty choice: a non-statistical meta-analysis of the literature. Acad Med 1995; 70: 620-41.

2. Rabinowitz HK, Diamond JJ, Markham FW, Hazelwood CE. A program to increase the number of family physicians in rural and underserved areas. JAMA 1999; 281: 255-60.

3. Rabinowitz HK, Diamond JJ, Markham FW, Rabinowitz C. Long-term retention of graduates from a program to increase the supply of rural family physicians. Acad Med 2005; 80: 72832.

4. Senf JH, Kutob R, Campos-Outcalt D. Wich primary care specialty? Factors that relate to a choice of family medicine, internal medicine, combined internal medicine-pediatrics, or pediatrics. Fam Med 2004; 36: 123-30.

5. Pugno P, Schmittling GT, McPherson DS, Kahn NB. Entry of US medical school graduates into family practice residencies: 1999-2000 and 3-year summary. Fam Med 2000; 32: 534-42.

6. Wright B, Scott I, Woloschuk W, Brenneis F. Career choice of new medical students at three Canadian universities: family medicine versus specialty medicine. CMAJ 2004; 170: 19204.

7. Ali B, Jones M. Do medical students want to become GPs? $\mathrm{Br}$ J Gen Pract 2003; 53: 241.

8. Tolhurst H, Stewart M. Becoming a GP. A qualitative study of the career interests of medical students. Aust Fam Physician 2005; 34: 204-6.

9. Lawson SR, Hoban JD. Predicting career decisions in primary care medicine: a theoretical analysis. J Contin Educ Health Prof 2003; 23: 68-80.

10. Dorsey ER, Jarjoura D, Rutecki GW. Influence of controllable lifestyle on recent trends in specialty choice by US medical students. JAMA 2003; 290: 1173-8.

11. Dorsey ER, Jarjoura D, Rutecki GW. The influence of controllable lifestyle and sex on the specialty choices of graduating U.S. medical students, 1996-2003. Acad Med 2005; 80: 791-6.

12. Lambert EM, Holmboe ES. The relationship between specialty choice and gender of U.S. medical students, 1990-2003. Acad Med 2005; 80: 797-802.

13. Newton DA, Grayson MS, Thompson LF. The variable influence of lifestyle and income on medical students' career specialty choices: data from two U.S. medical schools, 1998-2004. Acad Med 2005; 80: 809-14.

14. Block SD, Clark-Chiarelli N, Singer JD. Mixed messages about primary care in the culture of U.S. medical schools. Acad Med 1998; 73: 1087-94.

15. Whitcomb ME, Cullen TJ, Hart LG, Lishner DM, Rosenblatt RA Comparing the characteristics of schools that produce high percentages and low percentages of primary care physicians. Acad Med 1992; 67: 587-91.

16. Campos-Outcalt D, Senf J, Kutob R. A comparison of primary care graduates from schools with increasing production of family physicians to those from schools with decreasing production. Fam Med 2004; 36: 260-4.

17. Martini CJM, Veloski JJ, Barzansky B, Xu G, Fields SK. Medical school and student characteristics that influence choosing a generalist career. JAMA 1994; 272: 661-668.

18. Campos-Outcalt D, Senf J, Watkins AJ, Bastacky S. The effects of medical school curricula, faculty role models, and biomedical research support on choice of generalist physician careers: a review and quality assessment of the literature. Acad Med 1995;
70: $611-9$

19. Hearst N, Shore WB, Hudes ES, French L. Family practice bashing as perceived by students at a university medical center. Fam Med 1995; 27: 366-370.

20. Allen SS, Sherman MB, Bland CJ, Fiola JA. Effect of early exposure to family medicine on students' attitudes towards the specialty. J Med Educ 1987; 62: 911-7.

21. Beasley JW. Does teaching by family physicians in the second year of medical school increase student selection of family practice residencies? Fam Med 1993; 25: 174-5.

22. Osborn EHS. Factors influencing students' choices of primary care or others specialties. Acad Med 1993; 68: 572-4.

23. Campos-Outcalt D, Senf J. A longitudinal, national study of the effect of implementing a required third-year family practice clerkship or a department of family medicine on the selection of family medicine by medical students. Acad Med 1999; 74: 1016-20.

24. Bowman MA, Haynes RA, Rivo ML, Killian CD, Davis PH. Characteristics of medical students by level of interest in family practice. Fam Med 1996; 28: 713-9.

25. Herold AH, Woodard LJ, Pamies RJ, Roetzheim RG, Van Durme DJ, Micceri T. Influence of longitudinal care training on medical students' specialty choices. Acad Med 1993; 68: 281-4.

26. Lawson SR, Hoban JD, Mazmanian PE. Understanding primary care residency choices: a test of selected variables in the BlandMeurer Model. Acad Med 2004; 79: S36-9.

27. Kassebaum DG, Szenas PL, Schubert MK. Determinants of the generalist career intentions of 1995 graduating medical students. Acad Med 1996; 71: 197-209.

28. Rosenblatt RA, Andrilla CHA. The impact of US medical students' debt on their choice of primary care careers: an analysis of data from the 2002 medical school graduation questionnaire. Acad Med 2005; 80: 815-9.

29. Lynch DC, Newton DA, Grayson MS, Whitley TW. Influence of medical school on medical students' opinions about primary care practice. Acad Med 1998; 73: 433-5.

30. Campos-Outcalt D, Senf J, Kutob R. Comments heard by US medical students about family practice. Fam Med 2003; 35: 573-8.

31. Zinn WM, Sullivan AM, Zotov N, Peters AS, Connelly MT, Singer JD, Block SD. The effect of medical education on primary care orientation: results of two national surveys of students' and residents' perspectives. Acad Med 2001; 76: 355-65.

32. Markert RJ. Why medical students change to and from primary care as a career choice. Fam Med 1991; 23: 347-50.

33. Schafer S, Shore W, French L, Tovar J, Hughes S, Hearst N. Rejecting family practice: why medical students switch to other specialties. Fam Med 2000; 32: 320-5.

34. Nieman LZ, Foxhall LE, Chaung AZ, Cheng L, Prager TC. Evaluating the Texas Statewide Family Practice Preceptorship Program, 1992-2000. Acad Med 2004; 79: 62-8.

35. Morrison JM, Murray TS. Career preferences of medical students: influence of a new four-week attachment in general practice. Br J Gen Pract 1996; 46: 721-5.

36. Fincher RME, Lewis LA, Rogers LQ. Classification model that predicts medical students' choices of primary care or non-primary care specialties. Acad Med 1992; 67: 324-7.

37. Fincher RME, Lewis LA, Jackson TW and the Specialty Choice Study Group. Why students choose a primary care or nonprimary care career. Am J Med 1994; 97: 410-7.

38. Senf JH, Kutob R, Campos-Outcalt D. Wich primary care specialty? Factors that relate to a choice of family medicine, internal medicine, combined internal medicine-pediatrics, or pediatrics. Fam Med 2004; 36: 123-30. 